\title{
Bienestar animal aplicado a la producción bovina
}

\author{
Rossner, M.V.; Aguilar, N.M.; Koscinczuk, P. \\ Cátedra Bienestar Animal, Facultad de Ciencias. Veterinarias, UNNE, Sargento Cabral 2139, Corrientes (3400), \\ Argentina. Tel. 03783-425753. E-mail: mvrossner@hotmail.com.
}

\begin{abstract}
Resumen
Rossner, M.V.; Aguilar, N.M.; Koscinczuk, P.: Bienestar animal aplicado a la producción bovina. Rev. vet. 21: 2, 151-156, 2010. La preocupación acerca del bienestar animal ha ido en aumento, particularmente por la presión del público consumidor. Se entiende por bienestar al estado del individuo en relación a sus intentos de adaptarse al ambiente en un momento determinado. Al ser un concepto con bases científicas debe ser medido objetivamente. Por otra parte, la definición tiene algunas implicancias como la de ser una característica del animal, en lugar de algo que se le pueda brindar, así como variar dentro de un rango -desde muy bueno a muy malo- siendo malo cuando existen dificultades para mantener el control de las funciones o fallas en las tentativas de adaptarse al ambiente. En la cría y producción de bovinos existe una opinión generalizada que los sistemas extensivos son siempre buenos, porque mantienen a los animales lo más cerca posible del estado natural y que los sistemas intensivos son malos porque las libertades de elección y movimientos están restringidas. No obstante, en cualquier sistema productivo, extensivo o intensivo, pueden existir fracasos en la adaptación y consecuente estrés. Actualmente algunas organizaciones internacionales se han planteado como objetivo lograr que las nuevas tecnologías mejoren el bienestar sin producir mermas en la producción. Esto se puede alcanzar mediante el reconocimiento de los comportamientos naturales del bovino, teniendo en cuenta sus señales visuales, auditivas y olfativas, bases del manejo racional de los animales. Esta práctica no solo permite mejorar la relación humano-animal disminuyendo los signos de estrés, sino que también proporciona beneficios prácticos y económicos, generando efectos psicosociales positivos para el bienestar humano.
\end{abstract}

Palabras clave: bovino, manejo racional, producción de carne, sistemas intensivos.

\begin{abstract}
Rossner, M.V.; Aguilar, N.M.; Koscinczuk, P.: Animal welfare applied to cattle production. Rev. vet. 21: 2, 151-156, 2010. Concern about animal welfare has been increasing due to consumers' pressure. Animal welfare is defined as the individual state in its attempts to cope with the environment in a particular moment. Being a concept with scientific bases, it must be determined objectively. On the other hand, definition of welfare has some implications such as a characteristic inherent to the animal, contrary to something that may be offered or provided, as well as a variation within a range -from very good to very bad- being bad when there are difficulties to maintain normal functions or there is a lack in coping with the environment. There is a general opinion that extensive systems are always good because they allow animals to keep as close as possible to the natural state, and intensive systems are bad because freedom of choice and movement are restricted. But in any production system, failures in adaptation and consequent stress may be present. Nowadays, some global organizations pointed out new technologies that improve animal welfare without causing production losses. This can be reached by means of recognition of the natural behavior of cattle taking into account the visual, auditory and olfactory signals, being these baselines for rational handling. This practice not only improves the human-animal relationship by decreasing stress, but also provides practical and economical benefits generating psychosocial positive effects for human welfare.
\end{abstract}

Key words: cattle, rational handling, beef production, intensive systems. 


\section{La relación humano-animal}

El hombre siempre necesitó de los animales como fuente de comida, protección o compañía. Entre otras especies, los rumiantes se destacan por tener la capacidad de convertir alimentos de muy baja calidad como forrajes fibrosos y sub-productos agrícolas, en alimentos de alta calidad nutritiva, posibilitando el aprovechamiento de áreas donde las características del suelo o la topografía no permiten la agricultura mecanizada. El bovino es una de las especies que mejor se adaptó a vivir en domesticación. En los sistemas de pequeños productores el componente animal tiene múltiples propósitos, como alimentación, fuerza, trabajo, caja de ahorro y fuente de abono orgánico, entre otros ${ }^{21}$.

$\mathrm{Si}$ bien el vínculo humano-animal es tan viejo como la historia de la humanidad, lo que fue cambiando a lo largo del tiempo es la actitud del hombre hacia los animales. Inicialmente esta relación era la de aquel productor ganadero que conocía a cada uno de sus animales, atendiéndolos con un cuidado casi personalizado. Sin embargo, después de la segunda guerra mundial y debido a la gran hambruna y escasez de alimentos en Europa, comenzó el auge de los sistemas de producción intensivos. La necesidad de obtener alimentos ricos en proteínas de manera rápida y eficiente "industrializó" la producción, transformando a los animales en máquinas tecnológicas o instrumentos de producción de carne, leche $u$ otros productos para beneficio exclusivo del hombre, sin que éste tuviera en cuenta las necesidades propias de cada especie ${ }^{25}$.

La preocupación de los consumidores acerca del bienestar de los animales ha ido en aumento y cada vez son mayores las exigencias del público general respecto a la cría, transporte y faena de manera humanitaria ${ }^{20}$. No deberían resultar desconocidas las exigencias de organizaciones mundiales tales como la Oficina Internacional de Epizootias (OIE), la Organización Mundial de la Salud (OMS), la Organización de las Naciones Unidas para la Agricultura y la Alimentación (FAO) o los estándares sugeridos por la Unión Europea (UE). Es lógico esperar que aquellos países compradores que tienen y cumplen las normativas sobre el buen trato de los animales, exijan a los países proveedores iguales estándares ${ }^{8}$. De esta manera, surge que el conocimiento y respeto de la biología de los animales, además de permitir mejorar su bienestar, proporcionará mejores resultados económicos aumentando la eficiencia de los sistemas productivos y mejorando la calidad final del producto obtenido ${ }^{23}$.

\section{Fundamentos del bienestar animal}

Los primeros fundamentos sobre bienestar animal comenzaron a ser estudiados en el año 1965, cuando el Ministerio de Agricultura del Reino Unido reunió un comité de expertos para examinar las condiciones en que se encontraba el ganado mantenido en condiciones intensivas. Como estaba presidido por el profesor
Brambell, el documento por ellos emitido se conoce como el reporte Brambell. Su propuesta fue regirse por cinco principios básicos a los que llamaron las 5 libertades o necesidades.

Así, los animales deben tener libertad fisiológica (ausencia de hambre y sed), libertad ambiental (ausencia de incomodidad o malestar físico o térmico), libertad sanitaria (ausencia de dolor, enfermedad o lesiones), libertad psicológica (ausencia de miedo o angustia), libertad comportamental (posibilidad de expresar un patrón de comportamiento normal) ${ }^{14}$.

Estos cinco principios básicos defendidos por el Consejo de Bienestar Animal en Producción Animal del Reino Unido -Farm Animal Welfare Council- fueron adoptados en todo el mundo ${ }^{14}$. Deben cumplirse a través de la implementación de criterios adaptados a las diferentes especies y en diversas condiciones de producción. Varios países productores de carne bovina o sus derivados, actualmente cuentan con manuales de procedimientos que dictan los criterios a seguir para distintos tipos de manejo.

\section{Características del bienestar animal}

Si bien, el término bienestar se utiliza con frecuencia en el lenguaje coloquial, cuando queremos definirlo científicamente adquiere un significado más amplio, afectando no sólo al estado de salud del individuo, sino también a las necesidades, libertades, adaptación, control, sufrimiento, dolor, ansiedad y estrés, entre otros ${ }^{10}$.

Existen varias definiciones de bienestar animal pero es importante tener en cuenta un criterio esencial, el bienestar es una característica del animal y no algo dado por el hombre ${ }^{8}$. Es el estado del individuo en relación a sus intentos de adaptarse a su ambiente, en un momento determinado de su vida ${ }^{5}$.

Esta definición encierra varias características que hacen difícil su comprensión y, por lo tanto, su evaluación. Se han propuesto algunas implicancias de la definición de bienestar, donde se destacan los siguientes puntos:

I. El bienestar es una característica del animal, no es algo que le podamos dar o brindar. El bienestar del individuo surge de satisfacer sus necesidades propias y las de la especie. Los seres humanos no damos o brindamos bienestar, lo que podemos es aportar recursos para que el animal los utilice para intentar adaptarse al ambiente, satisfaciendo así sus necesidades. Estas necesidades son parte de la biología de los animales y es lo que un animal necesita para responder a un ambiente particular $u$ obtener un recurso ${ }^{7}$. Algunas necesidades se consideran básicas porque sin ellas la vida no es posible, como el agua o los alimentos. Otras, en cambio son menos urgentes, por ejemplo el esfuerzo que hace una vaca para cuidar a su ternero durante las primeras semanas post-parto. Ni la supervivencia de la vaca ni la del ternero se verían afectadas en el caso de realizar un destete precoz, sin embargo no escapa al observador 
la manera en que este procedimiento afecta a la calidad de vida de ambos ${ }^{6}$.

Es posible satisfacer las necesidades mediante modificaciones del ambiente, pero los estímulos ambientales son muy variables. Antes de definir si el medio es adecuado para un animal deberían ser evaluados todos los componentes de ese lugar (factores climáticos, alimento, agua, topografía, relaciones sociales dentro del grupo y con otras especies, como los perros y los seres humanos). Solo será considerado como apropiado cuando le permita al animal satisfacer sus necesidades ${ }^{2}$.

II. El bienestar varía dentro de un rango que abarca desde muy bueno a muy malo. Este rango variable es el resultado de una suma de factores entre los que se destacan el tiempo, la intensidad del estímulo, cómo el animal lo percibe, y la posibilidad de desarrollar una respuesta adaptativa. El término percepción implica una experiencia previa por parte del individuo y requiere de una elaboración cognitiva, donde el aprendizaje cobra particular importancia. Cada vez más, los estados mentales -sentimientos-de los animales son tenidos en cuenta. Se reconocen dos grandes grupos de sentimientos: los negativos y los positivos. Mientras que el sufrimiento es un sentimiento negativo, no placentero, que debe ser reconocido y prevenido siempre que sea posible ${ }^{8}$, los sentimientos positivos son aquellos que generan placer y constituyen un elemento significativo para entender el bienestar ${ }^{3,29}$.

Para algunos autores el bienestar depende solamente de las necesidades cognitivas, mentales o psicológicas de los animales, porque en general si éstas son cubiertas, los mismos alcanzarán a satisfacer sus necesidades físicas ${ }^{12}$.

III. El bienestar es malo cuando el animal tiene dificultades para mantener el control de sus funciones o falla en las tentativas de adaptarse al ambiente. Todavía hoy resulta más fácil determinar si el bienestar es pobre teniendo en cuenta las alteraciones multiorgánicas que surgen como consecuencia del estrés y la falta de adaptación. Algunos de estos ejemplos son los comportamientos estereotipados o la dificultad para reproducirse o amamantar a las crías. Las tendencias actuales apuntan a mejorar el bienestar generando emociones positivas. Se considera que unas pocas situaciones positivas tendrán un impacto más fuerte sobre el bienestar que muchas negativas ${ }^{3}$.

IV. El bienestar puede y debe ser medido cientificamente, no es subjetivo. La ciencia del bienestar es multidisciplinaria, por lo que basarnos en un solo aspecto no sería correcto. Para evaluar a un individuo debemos conocer su biología y el ambiente que lo rodea. Existen muchos indicadores de bienestar y su uso combinado garantiza una evaluación más completa de los individuos logrando objetividad ${ }^{24}$.

\section{Bienestar en sistemas intensivos y extensivos}

Existen diversas situaciones en las que el bienestar podría encontrarse más o menos comprometido, un ejemplo es el de los bovinos mantenidos en sistemas intensivos o a corral, debido a que en estas condiciones dependen completamente del sustento por parte del humano. Estos sistemas generan un nuevo desafío para la producción porque afectan fuertemente el bienestar de los animales.

Se define como sistema intensivo a aquel en donde se produce materia prima en cantidad, en el menor periodo de tiempo posible y en superficies reducidas ${ }^{21}$. En el caso particular de los bovinos, algunos ejemplos serían la recría de terneros de tambo y la recría o terminación en corrales de alimentación o feedlots (Figura 1).

La adaptación de un individuo al ambiente es el resultado de la interacción de su bagaje genético, el aprendizaje y dentro de éste, su experiencia previa ${ }^{30}$. Tomando como ejemplo a los bovinos, rumiantes gregarios y presa de carnívoros, que originalmente vivían en praderas, es de esperar que por su bagaje genético, estando en una pradera vivirán en condiciones de bienestar óptimo. Sin embargo, no siempre tendrán suficiente cantidad de comida y/o agua, o podrían ser atacados por carnívoros predadores o aves carroñeras, lo que afectará su bienestar. En cambio un bovino estabulado tendrá agua y comida disponible, refugio y protección, pero no siempre su bienestar será pleno, puesto que deberá enfrentarse con otras situaciones potencialmente negativas ${ }^{23}$.

Durante su evolución los bovinos aprendieron a obtener los recursos brindados por el hombre -comida,

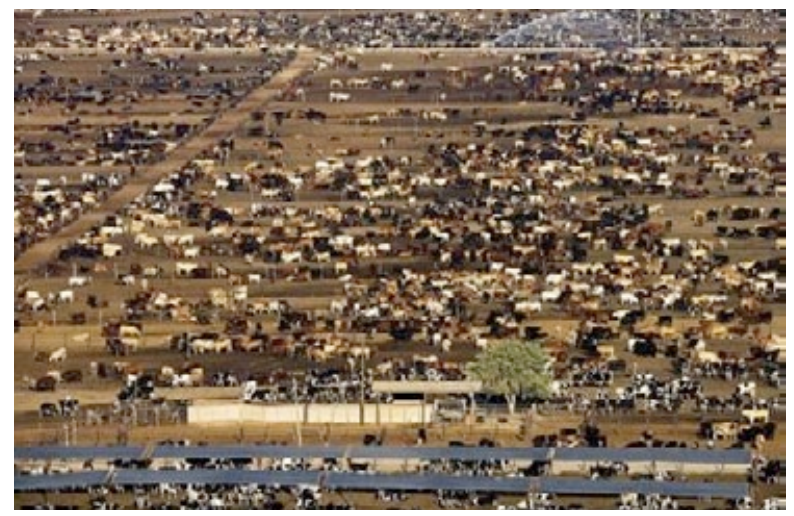

Figura 1. Cría de bovinos en sistema de producción intensivo.

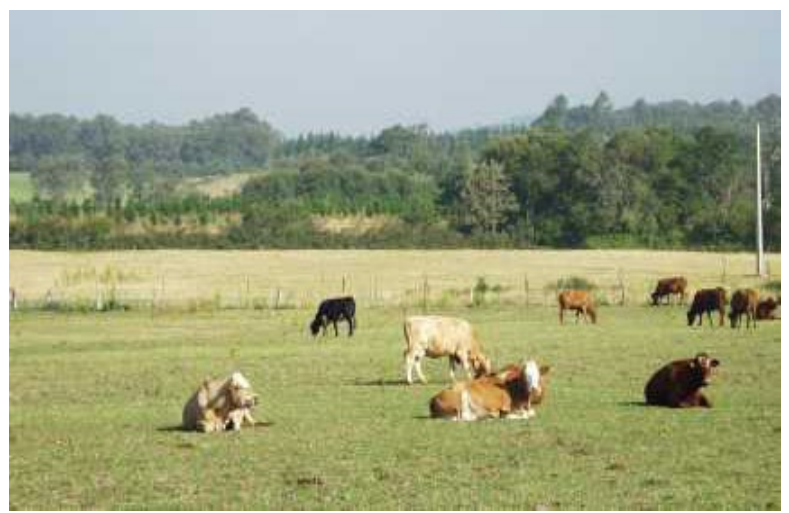

Figura 2. Cría de bovinos en sistema de producción extensivo. 
refugio, protección- hecho que favoreció la domesticación, sin embargo el maltrato al que pueden ser sometidos en los sistemas intensivos afecta seriamente al bienestar de los individuos debido a que estos sistemas muchas veces tienen en cuenta solo las necesidades físicas, pero no contemplan las necesidades comportamentales y menos aún las mentales ${ }^{12}$.

Pese a que no siempre ocurre, es opinión generalizada que los sistemas de producción extensivos (Figura 2) son siempre buenos porque permiten mantener a los animales lo más cerca posible al estado natural, con libertad de movimientos y manejo mínimo, y también se afirma que los sistemas intensivos o confinados son siempre malos porque están restringidas las libertades de elección, movimientos y expresión de comportamientos normales ${ }^{23}$.

\section{Estrés como fracaso de la adaptación}

En cualquier sistema productivo puede existir un fracaso de adaptación al ambiente generando una situación de estrés, el cual es considerado como un efecto ambiental que sobrepasa los sistemas de control del individuo ${ }^{8}$. La respuesta de estrés involucra tanto componentes fisiológicos como comportamentales ${ }^{27} \mathrm{e}$ inmunológicos, teniendo una base cognitiva muy fuerte ${ }^{26}$. La relación entre bienestar y estrés es evidente: siempre que hay estrés el bienestar se encuentra comprometido ${ }^{9}$.

Debemos considerar que las respuestas de estrés (Figura 3) tienen por finalidad mantener la homeostasis del medio interno y la perpetuación de la especie. Las situaciones de estrés crónico afectan a la producción de hormonas pituitarias. Si consideramos que las hormonas sexuales están gobernadas por el eje hipotálamo pituitario adrenal, fácil es reconocer que el estrés afecta a la reproducción ${ }^{26}$, por lo tanto cuando se evalúan los índices reproductivos, indirectamente se está evaluando el bienestar ${ }^{24}$. Por otra parte, el estrés también tiene impacto sobre la producción, de manera tan evidente

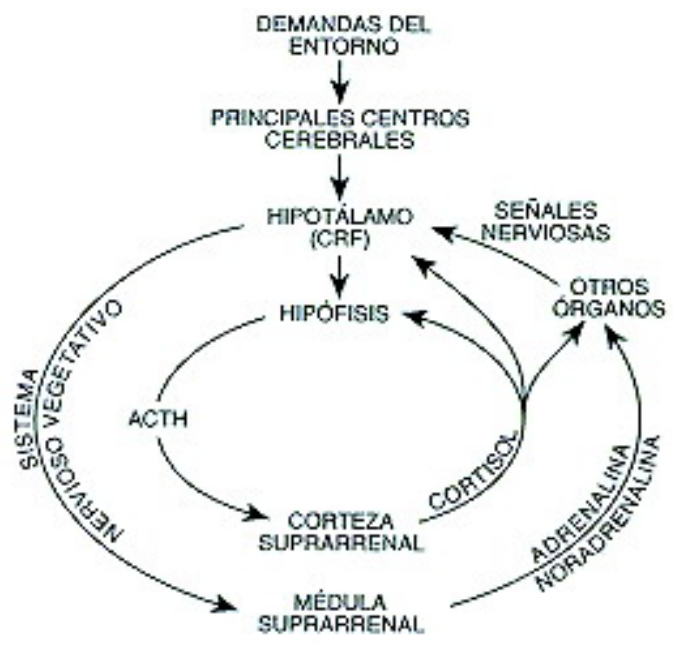

Figura 3. Eje neurohormonal del estrés. como las mermas en la producción de leche y ganancia diaria de peso vivo, o tan sutil como alterando la calidad de la carne (pH, terneza, sabor, color $)^{28}$.

Actualmente, las organizaciones internacionales tienen por objetivo lograr que la implementación de nuevas tecnologías pueda mejorar el bienestar sin producir mermas en la producción ${ }^{13,22}$. Las buenas prácticas de bienestar animal deberían incluir: prevención y tratamiento de enfermedades y lesiones; prevención y alivio del dolor, estrés y otros estados negativos; suministro de alimentación y condiciones de vida adecuadas a las necesidades y naturaleza de los animales; capacitación y entrenamiento de las personas que intervienen en el manejo de los animales ${ }^{22}$.

Muchos estudios comprueban que el buen manejo mejora la productividad de los animales ${ }^{18}$, por lo que es necesario modificar nuestra actitud con acciones, conocimiento y entrenamiento, utilizando el razonamiento para el manejo. El buen manejo es una tecnología de proceso simple, los operarios aprenden a través de la práctica y no requiere de grandes inversiones ni de mantenimiento. Se basa en el conocimiento científico de la reacción animal al manejo humano, en reemplazo del manejo basado en la fuerza física o la violencia ${ }^{1,15}$.

El manejo racional se basa fundamentalmente en aprovechar los comportamientos naturales de los bovinos, debido a que algunas características de su comportamiento son muy útiles, si se las tiene en cuenta a la hora de manejarlos: son animales gregarios, forman manadas y el grupo constituye su zona de seguridad. Son animales de presa que buscan alejarse de sus depredadores; sienten miedo cuando se los deja sin una salida visible ${ }^{15}$.

\section{Comportamiento bovino y manejo del rodeo}

Al ser gregarios los bovinos viven en grupos, siendo esta una estrategia adaptativa que ha evolucionado en muchas especies animales ${ }^{3}$. La vida en grupo tiene ventajas como defensa contra predadores e interacción sexual, así también desventajas como comportamientos agresivos debido a competencia por alimento, espacio y jerarquía ${ }^{11}$. Las relaciones sociales dentro de los grupos son mantenidas por amenazas y sumisión. Cada animal establece su espacio individual, importante para poder realizar movimientos básicos de echarse, levantarse, buscar alimento y agua. Los individuos actúan buscando su máximo beneficio y la interacción con sus pares afecta a todo el grupo. Los grupos estables pueden ser pensados como unidades de baja agresividad donde los animales no se mezclan con los de otros grupos y entre ellos se ha establecido una jerarquía de dominancia social ${ }^{19}$.

En condiciones de manejo intensivo de bovinos, al aumentar la densidad social existe una disponibilidad mínima de espacio por animal. Esto no permite a los subordinados escapar, hecho que aumenta la agresividad haciendo que la jerarquía de dominancia no funcione correctamente. Las interacciones entre los indivi- 
duos confinados pueden ser totalmente diferentes a las observadas en la naturaleza y no ser vistas en grupos de vida libre ${ }^{19}$.

Es primordial considerar que los bovinos tienen la capacidad de aprender, tanto cosas buenas como malas. La primera experiencia de un animal en un corral, con una persona o un equipo nuevo, debería ser lo más positiva posible. Si la primera vez se aplica un procedimiento muy doloroso o desagradable, puede hacerse difícil conseguir que el animal vuelva a entrar al mismo lugar fácilmente. Este tipo de reacción se da a través de un proceso de aprendizaje conocido como "condicionamiento o aprendizaje por asociación", mediante el cual el animal establece conexiones entre determinadas sensaciones y situaciones (involucrando personas o lugares). Si las sensaciones son negativas procurará evitar las situaciones asociadas a ellas, fugándose, luchando y dificultando el manejo. Los animales también aprenden por habituación, que es la manera en que se acostumbran a un tipo de manejo o de personas ${ }^{23}$.

Muchas veces al trabajar con el rodeo, nos comportamos como verdaderos predadores: galopamos, gritamos, agitamos e inclusive agredimos físicamente. En esas condiciones la reacción que podemos esperar es de miedo, favoreciendo que los animales nos eviten y/o ataquen. Pero el problema va más allá, debido a que el ganado tiene memoria y capacidad de reconocer personas o grupos de personas, las reacciones pueden ser cada vez más violentas, lo que resulta en experiencias negativas y un manejo más difícil ${ }^{17,23}$.

\section{La comunicación en los bovinos}

Mientras que en los seres humanos es importante la comunicación oral, en los bovinos adquieren mayor protagonismo las señales visuales, auditivas y olfativas (en ese orden de importancia). Las señales visuales son muy bien captadas debido al tipo de visión panorámica del vacuno, con un amplio ángulo de visión, por lo que pueden ver hacia atrás sin darse vuelta. Sin embargo, tienen un área ciega situada en la parte posterior del cuerpo, por detrás de la cola (Figura 4) ${ }^{16}$.

Este tipo de visión es común a todos los herbívoros y en sus antecesores constituyó un mecanismo útil para defenderse de los predadores. No obstante, es más imprecisa que la visión del ser humano por lo cual se distraen mucho al andar, particularmente cuando se encuentran con superficies con contrastes de luz y sombra u objetos sueltos en su camino ${ }^{16}$.

La posición del ser humano con respecto al bovino debería ser interpretada conociendo los conceptos de "distancia de fuga" y "punto de equilibrio" 16 . Distancia de fuga o huída es el trecho mínimo de aproximación de un extraño o predador capaz de ser tolerado por el animal antes de iniciar su fuga. Punto de equilibrio o de balance: son líneas imaginarias de referencia que cruzan perpendicularmente la paleta y el anca del animal; sirven como puntos de partida para iniciar o detener un movimiento. Para facilitar el manejo podemos ayudarnos con "banderas", las cuales reemplazan la fuerza física y actúan como señales visuales que orientan a los animales ${ }^{1,15}$.

Es fundamental el conocimiento del comportamiento normal de los bovinos para desarrollar la habilidad de reconocer señales de estrés y dolor, para poder tratarlos tempranamente. Al estar atentos a los cambios de comportamiento podemos detectar precozmente problemas que notaríamos muy tarde, por ejemplo, la disminución de la ganancia de peso de los animales ${ }^{24}$ . La aparición de comportamientos anormales nos indicaría que el bienestar de un individuo o grupo está comprometido. Podemos mencionar como ejemplo a las estereotipias, que son una secuencia relativamente invariable de movimientos que ocurren frecuentemente en un contexto particular y que no pueden ser considerados parte de los sistemas funcionales normales del animal. Estas forman parte del repertorio de comportamientos normales de los animales, pero si ocurren en forma prolongada o abarcan mucho tiempo durante el día, se tornan patológicas, reflejando que las condiciones son desfavorables para ese individuo ${ }^{4}$.

\section{Implicancias del buen manejo}

Considerando el concepto de bienestar animal podemos enumerar algunas acciones y beneficios al aplicarlo: las buenas técnicas de manejo mejoran el crecimiento y desarrollo de los animales, reduciendo dolor, miedo y reacciones fisiológicas de estrés provocadas por el manejo inadecuado. El suministro de dietas apropiadas y de suficiente agua potable contribuye a mantener la salud y productividad de los animales. Proporcionar condiciones de vida adecuadas a los bovinos puede disminuir la incidencia de comportamientos perjudiciales o anormales. Ambientes, instalaciones y equipos seguros y confortables pueden prevenir lesiones y pérdidas productivas. Al proporcionar espacio adecuado se evitan pérdidas productivas y muertes relacionadas con la superpoblación o hacinamiento. La mejor atención de los criadores o engordadores para con sus animales posibilita el diagnóstico precoz de enfermedades, de disminución de la producción y de

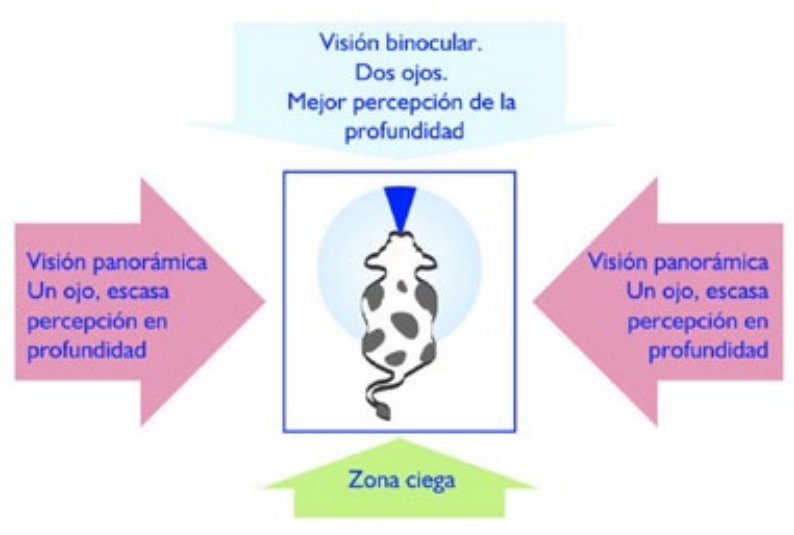

Figura 4. Diagrama de visión del bovino. 
problemas de comportamiento, aumentando en consecuencia la posibilidad de una intervención eficaz ${ }^{22}$.

\section{Colofón}

Además de los beneficios prácticos y económicos derivados de la comprensión del bienestar animal, puede concluirse que la interacción positiva con los bovinos puede proporcionar beneficios psicosociales importantes para el bienestar humano. Contribuir a la enseñanza de la ética del cuidado de los animales puede actuar como una fuerza de cohesión dentro de una familia, una comunidad o un negocio. La atención al bienestar animal puede tener beneficios muy amplios para las comunidades humanas, colaborando a la estabilidad de la región ${ }^{22}$.

\section{REFERENCIAS}

1. Aguilar NM, Paranhos MJ. 2009. Etología y bienestar animal conceptos a ser implementados en producción animal. Sistema de Información Técnica, INTA. On line: http://www.inta.gov.ar/benitez/info/indices/tematica/bienestar.htm.

2. Appleby MC. 1997. Life in a variable world: behaviour, welfare and environmental design. Appl Anim Behav Sci 54: 1-19.

3. Boissy A, Manteuffel G, Jensen M, Moe R, Spruijt B, Keeling L, Winckler C, Forkman B, Dimitrov I, Langbein J, Bakken M, Veissier I, Aubert A. 2007. Assessment of positive emotions in animals to improve their welfare. Physiol Behav 92: 375-397.

4. Broom DM. 1983. Stereotypes as animal welfare indicators. Indicators relevant to farm animal welfare. Curr Top Vet Anim Sci 23: 81-87.

5. Broom DM. 1986. Indicators of poor welfare. Br Vet $J 142$ : 524-526.

6. Broom DM. 1991. Needs and welfare of housed calves. In: New trends in veal calf production (Metz JH, Groenestein CM ed), Ed. Pudoc, Wageningen (The Netherlands), p. 23-31.

7. Broom DM. 1997. Welfare evaluation. Appl Anim Behav Sci 54: 21-23.

8. Broom DM. 2004. Bienestar animal. En: Etología aplica$d a$ (Galindo Maldonado F, Orihuela Trujillo A ed), Grupo Editorial Graphic-IFAW, México, p. 51-87.

9. Broom DM, Kirkden RD. 2004. Welfare, stress, behavior, and pathophysiology. En: Veterinary pathophysiology (Dunlop RH, Malbert CH ed), Blackwell, Iowa, p. 337369.

10. Broom DM, Molento CF. 2004. Bem-estar animal: conceito e questões relacionadas-revisão. Arch Vet Sci 9: 1-11.

11. De Vries T, von Keyserlingk M. 2006. Feed stalls affect the social and feeding behavior of lactating dairy cows. $J$ Dairy Sci 89: 3522-3531.

12. Duncan IJ, Petherick JC. 1991. The implications of cognitive processes for animal welfare. J Anim Sci 69: 50175022 .
13. Edwards JD. 2004. The role of the veterinarian in animal welfare. A global perspective. Proceedings of Global Conference on Animal Welfare, Paris, p. 27-32.

14. Farm Animal Welfare Council (FAWC). 1979. Press Statement. Publication on line: http://www.fawc.org.uk/ freedoms.htm.

15. Gimenez Zapiola M. 2006. Manual de buenas prácticas ganaderas, Ed. Cámara Argentina de Consignatarios de Ganado, Buenos Aires, p. 10-11.

16. Grandin T. 1997. The design and construction of facilities for handling cattle. Livestock Prod Sci 49: 103-119.

17. Grandin T. 1998. Review: reducing handling stress improve both productivity and welfare. The Professional Animal Scientist 14: 1-22.

18. Grandin T. 2003. Transferring results of behavioral research to industry to improve animal welfare on the farm, ranch and the slaughter plant. Appl Anim Behav Sci 81: 215-228.

19. Lindberg C. 2001. Group life. In: Social behavior in farm animals (Keeling LJ, Gonyou HW ed), CABI Pub., London, p. 37-54.

20. Manteca X. 1998. Neurophysiology and assessment of welfare. Meat Sci 49: 205-218.

21. Organización de las Naciones Unidas para la Agricultura y la Alimentación (FAO). 1997. Análisis de sistemas de producción animal: las bases conceptuales. Prod \& $S a$ nidad Anim 1: 1-80.

22. Organización de las Naciones Unidas para la Agricultura y la Alimentación (FAO). 2009. Capacitação para implementar boas práticas de bem-estar animal. Relatório do Encontro de Especialistas da FAO, Roma, p. 5.

23. Paranhos MJ. 2000. Ambiência na produção de bovinos de corte a pasto. Anais de Etologia 18: 26-42.

24. Parahnos MJ, Costa EV, Chiquitelli M, Rosa MS. 2002. Contribuição dos estudos de comportamento de bovinos para implementação de programas de qualidade de carne. Anais do XX Encontro Anual de Etologia, Natal (Brasil), p. 71-89.

25. Pompe V. 2005. The animal issue: diversity in values and thoughts. In: Animal bioethics: principles and teaching methods (Marie M, Edwards S, Gandini G, Reiss M, von Borell E ed), Wageningen Acad Publ, The Netherlands, p. 313-327.

26. Sapolsky RM, Romero LM, Munck AU. 2000. How do glucocorticoids influence stress responses? Integrating permissive, suppressive, stimulatory, and preparative actions. Endocr Reviews 21: 55-89.

27. Veissier I, Boissy A. 2007. Stress and welfare: two complementary concepts that are intrinsically related to the animal's point of view. Physiol Behav 92: 429-433.

28. Voisinet BD, Grandin T, Tatum JD, O'Connor SF, Struthers, JJ. 1997. Feedlot cattle with calm temperaments have higher average daily gains than cattle with excitable temperaments. J Anim Sci 75: 892-896.

29. Watanabe S. 2007. How animal psychology contributes to animal welfare. Appl Anim Behav Sci 106: 193-202.

30. Wechsler B, Lea SE. 2007. Adaptation by learning: its significance for farm animal husbandry. Appl Anim Behav Sci 108: 197-214. 